\title{
0
}

\section{PRESENTACIÓN: RETOS Y DESAFÍOS DE LA FORMACIÓN DEL PROFESORADO EN EL SIGLO XXI. UNA VISIÓN COMPARADA}

\author{
Presentation: Challenges of teacher education in the \\ $21^{\text {st }}$ Century. A comparative approach
}

\author{
Amalia Ayala* y Antonio Luzón **
}

\section{INTRODUCCIÓN}

Abordar la coordinación de un número monográfico sobre la formación del profesorado en el siglo XXI, desde una perspectiva comparada ha entrañado la gratificante tarea precisamente de coordinar las aportaciones y agendas con numerosas personas del continente europeo y americano, que son las que finalmente lo han hecho posible. En nuestro contexto de referencia más inmediato, el del panorama universitario español, nos situamos precisamente en el momento que toca hacer balance de un nuevo modelo de formación de jóvenes graduados, futuros docentes de Educación Infantil, Primaria y Secundaria, ajustada a los nuevos planes de formación de profesorado conforme al proceso de convergencia del Espacio Europeo de Educación Superior (EEES). Parece una obviedad afirmar lo que todos sabemos, que una de las piezas claves para mejorar el sistema educativo es el profesorado, de ahí que su formación, tanto inicial como continua, sea uno de los ejes en los que gravitan las políticas educativas y de hecho figure en las agendas de las organizaciones supranacionales. Así, la OCDE (2009) en una publicación titulada, en su versión en castellano, Política de educación y formación: Los docentes son importantes. Atraer, formar y conservar a los docentes eficientes, afirma de forma contundente que: "Los buenos docentes son la columna vertebral de todo sistema educativo. Por tal razón, los gobiernos constantemente buscan

\footnotetext{
* Universidad de Murcia (España).

** Universidad de Granada (España).
} 
políticas que los ayuden a seleccionar y conservar a los mejores". No en vano en una publicación posterior, también de la OCDE y editada por Andreas Schleicher ${ }^{1}$ (2012), titulada Preparing Teachers and Developing School Leaders for the 21st Century Lessons from around the World, se vuelve a insistir en la idea de la necesidad de introducir reformas en los planes de formación del profesorado con el objetivo de preparar mejor a las jóvenes generaciones ante las demandas socioeconómicas que plantea un mundo en constante cambio y de forma más cada vez más rápida. Al mismo tiempo que Schleicher (2012) formula la necesidad de estas reformas, también plantea qué necesidades o requisitos han de reunir los programas de formación docente para garantizar la preparación de habilidades (skills) o competencias básicas de niños y jóvenes en sociedades cada vez más complejas y diversas. O bien, como se aboga en otro reciente informe de la OCDE (2013) titulado Teachers for the 21 st Century Using Evaluation to Improve Teaching de una valoración de la función docente a través de la evaluación.

De modo que, en este momento de realidades macro con una nueva reforma del sistema educativo, de rediseño curricular, de cuestionamiento de la formación docente y su adecuación al momento histórico que vivimos, en tiempos de reflexión de cómo aprender más y enseñar mejor, pensamos que es tiempo también de detenerse y pensar, lo que a nivel micro nos puede señalar algunas luces sobre las muchas sombras de un proceso (sobre todo para el caso español), al que no ha acompañado precisamente las condiciones económicas materiales y humanas. Creemos pertinente en esta presentación dar cabida al planteamiento que se hicieran Jesús Romero y Alberto Luís (2006) hace algunos años en el monográfico "Formación crítica del profesorado y profesionalidad democrática” en Con-ciencia Social (10) “¿sirve la formación del profesorado para mejorar la educación?”. Y es que no es ninguna novedad que la formación del profesorado se haya erigido y enarbolado en estas últimas décadas como uno de los factores condicionantes de la calidad en nuestros sistemas educativos. En este proceso de cambios y reformas continuas que afectan a más de cuatro millones de docentes en la UE, tampoco el panorama internacional despeja dudas sobre lo que acontece, como bien apunta Alejando Tiana en su contribución en este mismo monográfico. La Revista Interuniversitaria de Formación del Profesorado, (68) ${ }^{2}$ en 2010 se hizo eco de esta problemática y publicó un número monográfico titulado "Reinventar la profesión docente. Nuevas exigencias y escenarios en la era de la información y la incertidumbre", poniendo de manifiesto la insatisfacción generalizada con la formación de los actuales docentes ya que "tiene que ver con un modelo ya obsoleto, pero resistente, de supuesta aplicación diferida y directa de la teoría a la práctica" (p.12). Desde otra perspectiva no menos intersante, Inmaculada Egido (2011), también percibe este interés por las reformas en la formación del profesordo y en un artículo titulado "Cambios y dilemas en la formación del profesorado (1961-2011). Cincuenta años de historia de España en perspectiva europea" y publicado en Tendencias Pedagógicas (nº 18), analiza las reformas más notables en la formación docente en los últimos cincuenta años en nuestro país desde una perspectiva comparada. En una línea similar se encuadra el trabajo de compilación de Leoncio Vega (2011) que tiene por título Gobernanza y políticas de formación inicial de profesores en la Europa Mediterránea, donde plantea si las políticas de formación docente se dirigen más bien

1 Subdirector de Educación en la OCDE desde febrero del 2012, y Coordinador del Programme for International Student Assessment (informe PISA).

${ }^{2}$ Para una consulta más amplia, se puede consultar en:

http://www.aufop.com/aufop/revistas/indice/impresa/151 
hacia una síntesis de convergencias y divergencias de procesos de formación o nos situamos en otros parámetros de estudios diferentes. Para ello se detienen a analizar los casos de formación inicial de profesorado en España, Italia, Portugal y Francia.

Así pues, las críticas y autocríticas que inciden en ese inusitado interés por la formación de los docentes, sigue estando de actualidad, tanto a escala local como supranacional, en lo que parece más bien un interés de carácter discursivo. La Comisión Europea ${ }^{3}$ (2012) en un documento titulado "Supporting the Teaching Professions for Better Learning Outcomes. Rethinking Education: Investing in skills for better socio-economic outcomes", enfatiza la importancia de las habilidades, del "saber hacer" en la formación docente para la obtención de mejores niveles de bienestar, sobre todo de carácter socioeconómico. Fue también la Comisión Europea (2006) a través de la red Eurydice $^{4}$, la que alertara del gran número de iniciativas en muchos países de Europa referentes a la evaluación de la calidad y eficacia de los sistemas de educación y formación, siguiendo uno de los objetivos marcados en la denominada estrategia de Lisboa. Precisamente una de esas iniciativas estaba relacionada con la "calidad de la formación del profesorado europeo". Posteriormente el Consejo Europeo (2007) $)^{5}$ emite una serie de consideraciones a tener en cuenta por los respectivos países sobre la mejora de la calidad de la formación del profesorado, destacando especialmente el profesorado de altas cualificaciones. Por tanto, volvemos a una lógica discursiva que oscurece el campo de visión, la necesidad de abordar la formación que necesita un buen profesor. Antonio Nóvoa (2009:206), con buen criterio expone que al margen de los criterios de carácter conductual e instrumental que infieren determinados posicionamientos que han regulado la formación docente durante la segunda mitad del siglo XX, las nuevas orientaciones en este ámbito se han limitado a enumerar una serie de competencias para definir el conocimiento (saber), las habilidades (saber hacer) y las actitudes (saber ser) de los futuros docentes (PERRENOUD, 2010). Más allá de la conveniencia o adaptación de los modelos de formación inicial clásicos, simultáneos o consecutivos, Nòvoa (2009:208) plantea que la formación del "buen docente" hay que construirla desde la práctica, es decir centrada en el aprendizaje del alumnado, desde un enfoque del trabajo docente, teniendo como referencia el trabajo escolar. Por otra parte, desde los organismos supranacionales se incide en políticas con orientaciones que incluyen una normatividad pseudoregulatoria, con un discurso performativo, basado principalmente en resultados, que a juicio de Bolívar (2013: 63), erosionan la implicación docente y al mismo tiempo debilitan su compromiso.

En una reciente publicación, Darling-Hammond junto a Lieberman (2012) también constataban la evidencia de que el profesorado constituye el elemento más importante del sistema educativo, pero se cuestionaban las estrategias o dispositivos necesarios para la creación de docentes de alta calidad en el mundo de hoy. De este modo dichas autoras aluden a las comparaciones internacionales para comprender los ajustes y cambios que se están produciendo en los diferentes países de cara a satisfacer las demandas globales. En esta línea, también Eurydice

\footnotetext{
${ }^{3}$ Puede consultarse en: http://ec.europa.eu/education/news/rethinking/sw374 en.pdf

${ }^{4}$ Eurydice (2006): La evaluación de la calidad de la formación del profesorado europeo. Bruselas. Disponible en: http://www.eurydice.org

${ }^{5}$ Consultar en http://eur-lex.europa.eu/LexUriServ/LexUriServ.do?uri=OJ:C:2007:300:0006:0009:ES:PDF
} 
$(2013)^{6}$, acaba de publicar un Informe titulado Key Data on Teachers and School Leaders in Europe (Cifras clave sobre el profesorado y los directores de centro en Europa) donde se presenta de forma descriptiva el panorama actual del profesorado europeo.

La cuestión estriba en proporcionar nuevos docentes de alta calidad, pero se carece de información sobre las políticas y prácticas que se están desarrollando. Precisamente desde el pequeño observatorio que nos ha brindado la Revista Española de Educación Comparada queremos contribuir a ese conocimiento, referido especialmente al continente europeo y latinoamericano.

\section{ESTRUCTURA}

Iniciamos este número con la contribución de Antonio Viñao, catedrático de la Universidad de Murcia y destacado especialista en Historia de la Educación, que aporta una indiscutible y valiosa perspectiva histórica a la formación inicial del profesorado en España, concretamente centrada en la Educación Secundaria, nivel que arrastra grandes carencias en su formación pedagógica. El artículo titulado "Modelos de formación inicial del profesorado de Educación Secundaria en España: análisis histórico", examina, analiza e identifica desde una perspectiva histórico-comparada seis modelos de formación inicial del profesorado de educación secundaria que han existido en España, partiendo desde los años treinta del siglo XIX hasta la actualidad, momento en el que queda descrito el sexto modelo, consecutivo, dentro de la estructura del proceso de Bolonia, con la categoría de titulación de postgrado, denominado Máster para el profesorado de Educación Secundaria. Como señala Viñao, a lo largo del tiempo se cambia la nominación y el estatuto académico, pero no el modelo formativo. Es más, el modelo que la lógica formativa aconseja se resiste a implantarse por diversos y variados motivos entre los que el autor destaca el equilibrio de poder entre los cuerpos afectados, mantenimiento temporal de vías nuevas y tradicionales, de acuerdo entre Comunidades Autónomas...No obstante, sobrecogen algunas de sus reflexiones de corte lampedusiano "...para que nada sustancial cambie es necesario que parezca que todo cambia".

La contribución de Alejandro Tiana, catedrático de la Universidad Nacional de Educación a Distancia (UNED) y Secretario General de Educación (2004-2008), titulada "Los cambios recientes en la formación inicial de profesorado en España: una reforma incompleta" nos sitúa, desde su privilegiado observatorio, en la necesidad de desarrollar políticas integradas que han de incluir, entre otros muchos factores fundamentales, la formación docente. Su contribución tiene el mérito de hacer un recorrido por las diferentes políticas de esta última década en el contexto internacional, sobre todo a partir de la estrategia de Lisboa. Se detiene y analiza los variados informes y actuaciones que en relación a la formación docente han ido elaborando organismos como la OCDE (2005; TALIS, 2009; Cumbre Internacional sobre la Profesión Docente, 2011...), Comisión Europea (2010) y la unidad Eurydice (2002-2005), al igual que consultoras como McKinsey \& Company (2007, 2010). Tras una descripción del panorama general, constata que muchos de estos informes

\footnotetext{
${ }^{6}$ El texto completo se puede consultar en Eurydice: http://eacea.ec.europa.eu/education/eurydice/documents/key_data series/151EN.pdf
} 
internacionales no llegaron a tiempo para inspirar la reforma legislativa y normativa desarrollada en España desde 2005 hasta 2007, en el proceso de regulación y elaboración del modelo formativo español, al amparo del EEES.

El texto de Pierre-Louis Gauthier titulado "France: la formation des enseignants en échec" sobre la formación del profesorado en Francia, nos permite, desde su trayectoria profesional al servicio de la administración educativa, introducirnos en unos sugerentes epígrafes que ofrecen una visión contextual de los numerosos desequilibrios sociales y su repercusión en materia educativa en tiempos de crisis de la educación a escala global. Describe con suma maestría la inadaptación de la estructura escolar a la nueva situación socioeconómica, poniendo en entredicho la propia capacidad del sistema para responder a las nuevas exigencias que la sociedad plural plantea. Al mismo tiempo Gauthier, desde una perspectiva crítica, invita a la reflexión sobre el paulatino declive del imperio escolar francés en el que se evidencia cómo la fractura escolar sobrepasa en amplitud a la fractura social y muestra cómo una escuela históricamente concebida como instrumento de garantía de igualdad se resiente ante la realidad de los recortes presupuestarios que han acompañado las políticas educativas francesas más recientes, sobre todo centradas en el periodo de gobierno de Sarkozy con la supresión de 80.000 puestos escolares en cinco años. Además, en este artículo, Pierre Louis Gauthier realiza un amplio recorrido histórico de la formación inicial de profesorado en Francia desde la supresión de las Escuelas Normales hasta los IUFM; y de éstos a las Écoles Superiures du Professorat et de l'Éducation (ESPE), destinados a reemplazar a los denostado IUFM's. Analiza con sumo detalle el compromiso de no disociarse de los grandes proyectos de los maestros republicanos retomando su vocación original de conquista de la igualdad.

No podía faltar el caso de Alemania en este recorrido por los retos y desafíos que atraviesa la formación inicial del profesorado en el continente europeo. Una aportación brillante, coherente y clara la que han realizado Hans-Georg Kotthoff, catedrático de la Universidad Pedagógica de Friburgo y Ewald Terhart, catedrático de la Universidad de Münster, titulada "New solutions to "old" problems? Recent reforms in Teacher Education in Germany". Su punto de partida es la identificación de estructuras y discusiones relativas a la formación docente en Alemania con tres procesos que se entrecruzan: el proceso Bolonia, la profesionalización y la estandarización de la formación docente, en un recorrido que desde mediados de los años noventa acompaña las reformas diferenciadas en los dieciséis Länders. Los autores analizan las diferentes y recientes reformas acontecidas en cuanto formación docente desde una perspectiva comparada e internacional, pero sin olvidar el contexto histórico en el tradicional sistema educativo germano, entrando a estudiar los problemas estructurales y también las nuevas evidencias empíricas que han modificado por un lado los modelos y conceptos tradicionales y por otro han iniciado las recientes reformas. Diferencias pues que se constatan entre los modelos de formación docente en los niveles de primaria y secundaria con estructuras horizontal y vertical respectivamente, teniendo en cuenta los cambios en los respectivos Länders.

Tampoco podía faltar en este monográfico una visión pormenorizada de la formación inicial docente en Inglaterra. Ann Childs e Ian Menter, de la Universidad de Oxford, aportan un lúcido y sugerente texto titulado, "Teacher Education in $21^{\text {st }}$ Century England - A Case Study in Neoliberal Public Policy”, donde examinan y analizan detenidamente la formación docente, especialmente la 
inicial, donde ponen de manifiesto las sucesivas regulaciones gubernamentales desde 1984. En su análisis del caso de la formación docente en Inglaterra, tanto Childs como Menter, muestran una trayectoria global de las políticas que han sustentado las sucesivas regulaciones de la formación de los futuros docentes, justamente desde una perspectiva neoliberal en un sistema mercantilizado. Este rumbo u hoja de ruta trazada durante la etapa de Margaret Thatcher no se ha desviado, a pesar de los cambios de gobierno ocurridos a partir de 1997 con los gobiernos de corte laborista de Tony Blair y posteriormente de Gordon Brown. Tampoco este rumbo, dibujado treinta años atrás, ha cambiado con el actual gobierno de coalición de los partidos conservador y liberal-demócrata, desde 2010. En sus dos partes, el artículo explora la evolución de las políticas de formación docente desde la década de los ochenta y una segunda parte donde se aproxima a la problemática que afecta a la formación y profesión docente en la actualidad, donde la calidad de la enseñanza tiende a debilitarse debido entre otras cosas a la tensión permanente entre la libertad de acción y mayor regulación y restricciones. Por una parte se liberaliza el sistema de formación pero por otra se incrementan los mecanismos de control y restricciones a la creatividad y a la autonomía. Sin embargo, Popkewitz (1997: 104), afirmaba ya hace algunos años que sería una equivocación pensar que una reforma aporta más autonomía y flexibilidad al profesorado frente a las fuerzas del mercado, ya que estos cambios no ponen fin a los problemas de regulación sino que los reconstruyen. De ahí esta reconfiguración constante donde la libertad se convierte asimismo en elemento de control, debilita la contribución de las universidades y desestabiliza y debilita su papel en cuanto a la formación docente.

Era ineludible examinar y analizar también el caso de la formación docente en Finlandia, sobre todo ante el éxito de su sistema educativo en las evaluaciones de PISA, donde las comparaciones internacionales miran a sus escuelas y una de las claves apunta a la formación del profesorado. Hannele Niemi, catedrática de la Universidad de Helsinki y con una amplia experiencia investigadora en el campo de la formación docente, colabora en este número con un artículo titulado "The Finnish Teacher Education - Teahers for Equity and Professional Autonomy", en el que describe las principales características del sistema educativo finlandés y la apuesta por la excelencia en la formación del profesorado. Hannele Niemi, es conocida por la obra publicada en nuestro país por el Ministerio de Educación (2008), titulada Aprender de Finlandia. La apuesta por un profesorado investigador y alguna que otra colaboración en Revista de Educación. En este artículo, Niemi examina con suma precisión las características básicas y los programas nacionales del sistema educativo, basado durante los últimos treinta años, principalmente en dos principios que vertebra a todo el sistema: la equidad y el aprendizaje permanente. Realiza una somera descripción del proceso de formación docente, ya que desde 1979, todo el profesorado ha tenido que cursar cinco años, incluso reforzada a nivel de Máster con el proceso de Bolonia. Para Niemi, el principio fundamental de la formación docente es educar a los profesores como profesionales autónomos, donde prima la alta calidad de su formación, haciendo especial hincapié en la cultura profesional basada en la investigación científica, la integración de la teoría y la práctica y un código social y moral de la profesión docente. Atrae a gran número de candidatos talentosos por lo que existe un alto nivel de selección entre los estudiantes a convertirse en futuros profesores. La autora también plantea en este texto los principales desafíos a los que se enfrenta la formación docente en Finlandia. El desarrollo de una cultura de enseñanza basada en un aprendizaje más activo, el desarrollo de competencias para una escuela multicultural y el uso pedagógico y 
aplicaciones de las nuevas tecnologías constituyen grandes retos para la formación docente y cultura escolar en Finlandia.

Otro caso del continente europeo que no podía faltar en este número era Italia, sobre todo por estar en pleno proceso de reforma a todo lo largo de 2012. Los profesores de la Universidad de Roma-2 "Tor Vergata", Carlo Cappa, Orazio Niceforo y Donatella Palomba describen y analizan meticulosamente los cambios que se han ido produciendo en la formación inicial del profesorado en Italia, y sobre todo como consecuencia del proceso de Bolonia. El texto, que reproducimos en italiano, "La formazione iniziale degli insegnanti in Italia", se divide en tres partes. Un primer apartado contempla aquellos elementos históricos a tener en cuenta para comprender la evolución del proceso de la formación inicial del profesorado italiano, ya que no siempre ha sido formado en las universidades y la diferencia de modelo formativo del profesorado de la escuela infantil o primaria y el profesorado de la escuela secundaria. La segunda parte analiza los cambios acaecidos en la última década, la academización de la formación docente, tanto en lo que respecta a la educación primaria que pasa a depender de la Universidad (laurea in Scienze della Formazione Primaria), y la creación de la Scuole di Specializzazione per l'Insegnamento Secondario (SSIS) hasta la supresión de esta última, en 2009.

En la tercera parte del artículo, los autores presentan el marco actual de la formación inicial del profesorado en Italia, especialmente la del profesorado de secundaria, que a diferencia del resto del profesorado desde 2010 se han ido produciendo importantes novedades. Las Scuole di Specializzazione per l'Insegnamento Secondario (SSIS) se han sustituido por una tipología de formación práctica, denominada Tirocinio Formativo Attivo (TFA), que está siendo implementada por las universidades. El artículo concluye con un breve balance de la experiencia del Tirocinio Formativo Attivo, donde se expone de forma somera algunos problemas suscitados como consecuencia de la puesta en práctica de esta modalidad formativa.

Para darle un carácter más comparado, si cabe, al número monográfico dedicado a la formación incial docente, nos pareció intereasante aportar una colabración que abordase un planteamiento integrado de la formación del profesorado en la Unión Europea. La aportación de Jesús Manso y Javier Valle, miembros del Grupo de Investigación sobre Políticas Educativas Supranacionales de la Universidad Autónoma de Madrid titulada "La formación inicial del profesorado de secundaria en la Unión Europea", cubre con creces este objetivo. El artículo examina y afronta de manera exhaustiva el incremento de medidas, así como propuestas, relacionadas directamente con la formación inicial del profesorado desde la Unión Europea. Buscando una lógica de armonización o integración de políticas entre los diferentes países, los autores, bajo una perspectiva comparada, tienden a identificar tendencias, modelos, que permitan reflexionar y comprender mejor las políticas educativas de la Unión Europea y las líneas derivadas de las mismas en torno a la formación docente, susceptibles de los cambios o adaptaciones locales de cada país en función de su contexto y características singulares.

También bajo este prima integrador y enfoque comparado, nos parecía enriquecedor conocer ampliamente la visión latinoamericana. Una gran conocedora de las políticas de formación del profesorado en Latinoamérica como Denise Vaillant, profesora del Instituto de Educación de la 
Universidad (Uruguay), aporta un sugerente e interesante artículo titulado "Formación inicial del profesorado en América Latina: dilemas centrales y perspectivas", donde evidencia un interés generalizado una mayoría de países durante las últimas décadas por la formación docente. El gran dilema que plantea la autora es que la profesión docente en América Latina no logra retener a los buenos docentes, por lo que constituye uno de los mayores retos a resolver en la región. Sin embargo, a juicio de Vaillant, es una problemática aislada que precise de una solución específica, sino que es de más amplio alcance y ha de analizarse a la luz de una amplia gama de factores que definen a la profesión docente en América Latina. No obstante, se constata que en algunos países la mejora de la formación inicial del profesorado es factible cuando existe voluntad política, aunque la situación en otros casos es más compleja y requiere elevar el prestigio social de los docentes propiciando mejores oportunidades y mayores incentivos económicos y simbólicos.

Este extenso número concluye, a modo de epílogo, con un memorable artículo dedicado a la contribución de José Manuel Esteve al estudio e investigación sobre la profesión docente. La difusión de su trabajo en los ámbitos comparados ha contado tanto con las referencias documentales europeas, en las que el profesor Esteve fue actor principal, como con el recuerdo y respeto de una escuela en la que ilustres miembros de la comunidad científica comparada española han tenido mucho que ver, como es el caso de la profesora Ángela Caballero de la Universidad de Málaga. El autor de este merecido homenaje es Julio Vera, también de la Universidad de Málaga, compañero y amigo del profesor Esteve, que expone al detalle y con suma delicadeza los rasgos de su personalidad, su contribución, así como los hitos más relevantes de su vida profesional y sus principales publicaciones desde sus comienzos como profesor universitario hasta su fallecimiento, ocurrido en 2010. Nos parecía el mejor broche para cerrar un número monográfico que pretende mostrar, contribuir y enriquecer el debate actual sobre la formación docente, incidiendo especialmente en la formación inicial, así como las políticas educativas subyacentes, desde una perspectiva crítica y comparada.

\section{REFERENCIAS}

BOLÍVAR, A. (2013). La lógica del compromiso del profesorado y la responsabilidad de la escuela. Una nueva mirada. Revista Iberoamericana sobre Calidad, Eficacia y Cambio en Educación, 11 (2), pp. 61-86. (http://www.rinace.net/reice/numeros/arts/vol11num2/art3.pdf)

DARLING-HAMMOND, L. \& LIEBEREMAN, A. (2012) (eds.). Teacher Education around the World. Changing policies and practices. (London and New York, Routledge).

EGIDO GÁLVEZ, I. (2011). Cambios y dilemas en la formación del profesorado (1961-2011). Cincuenta años de historia de España en perspectiva europea, Tendencias Pedagógicas, 18; pp. 33-50.

EURYDICE (2013). Key Data on Teachers and School Leaders. (Eurydice Report. Luxembourg: Publications Office European Union). (http://eacea.ec.europa.eu/education/eurydice/documents/key_data_series/151EN.pdf) 
-(2006). La evaluación de la calidad de la formación del profesorado europeo. (Bruselas, Eurydice Unidad Europea).

JAKKU-SHIVONEN, R. y NIEMI, H. (2008). Aprender de Finlandia. La apuesta por un profesorado investigador (Madrid: Ministerio de Educación).

NÓVOA, A. (2009). Para una formación de profesores construida dentro de la profesión, Revista de Educación, 350; pp. 203-2018.

OCDE (2009). Política de educación y formación: Los docentes son importantes Atraer, formar y conservar a los docentes eficientes. (París, OCDE).

OCDE (2013). Teachers for the 21st Century. Using Evaluation to Improve Teaching. (OECD Publishing).

PERRENOUD, Ph. (2009). Diez nuevas competencias para enseñar: invitación al viaje. (Barcelona: Graó).

POPKEWITZ, T. (1997). La profesionalización, el gobierno del profesor y el conocimiento académico: Algunas notas comparativas. Revista Interuniversitaria de Formación del Profesorado, 29; pp. 89-109.

REVISTA INTERUNIVERSITARIA DE FORMACIÓN DE PROFESORADO (2010). "Reinventar la profesión docente. Nuevas exigencias y escenarios en la era de la información y la incertidumbre", (Monográfico), 68 (24,2). (http://www.aufop.com/aufop/uploaded_files/congresos/1296516384.pdf)

ROMERO, J., LUIS, A. y Otros (2006). La formación del profesorado y la construcción social de la docencia, Con-ciencia Social, 10; pp. 13-68.

VEGA GIL, L. (2011). Gobernanza y políticas de formación inicial de profesores en la Europa Mediterránea. (Valencia, Tirant Lo Blanch).

SCHLEICHER, A. (2012). Preparing Teachers and Developing School Leaders for the 21st Century Lessons from around the World. (OECD Publishing). 